\title{
Joint Design for a Hyper-Redundant Serial Manipulator
}

\author{
Mark Alonso \\ McGill University \\ mark.alonso@mail.mcgill.ca \\ Mark Stephenson \\ McGill University \\ mark.stephenson@mail.mcgill.ca
}

\author{
Afaan Naqvi \\ McGill University \\ afaan.naqvi@mail.mcgill.ca
}

\begin{abstract}
We propose a joint design for a low-cost, light-weight, hyper-redundant serial manipulator. The proposed design consists of two rigid end plates connected by a universal joint; two sets of agonist/antagonist linear actuator pairs allow independent 2-DOF control. In tethered configuration, power and control signals are delivered to the robot by wire; the proposed control scheme is an open-loop relay system. The actuators consist of approximately helical coils of shape memory alloy wire. Current is alternately directed (at a frequency of $10 \mathrm{~Hz}$ ) through the members of each actuator pair; joint deflection is achieved by varying the respective duty cycles.
\end{abstract}

\section{Introduction}

Traditional rigid manipulators have limited flexibility and can both cause and sustain considerable damage during collisions. It is thought that hyper-redundant serial manipulators could overcome these deficiencies; such a "robotic tentacle" would be particularly attractive in irregular environments, or those shared by humans. It would also have greater fault tolerance - if a joint is locked or is loose, dynamic properties can be used to control the tentacle.

The range of potential applications for this technology is wide: in the space domain, a robotic tentacle could be used for manipulation, inspection, assembly and locomotion. A tentacle does not need special fixtures in order to move around; consequently, it could be deployed in an ordinary, low-cost environment containing only hand-holds for people. Furthermore, there are a number of possible terrestrial applications: as an inspection device in hazardous and irregular environments, for example, or as a search and rescue tool in collapsed buildings.

The work presented in this article was carried out at McGill University as a capstone design project, in partial fulfillment of the requirements for the B.Eng. (Mech.) degree. The project was proposed and funded by our client at the Canadian Space Agency, Mr. Leo Hartman. The desired outcome is a functioning prototype, to be used as a test bed to explore intelligent control systems for an innovative mobile platform; further engineering constraints are described in Section 2 below.

We were attracted to this unique and challenging project for several reasons. We wanted to take advantage of the opportunity to work with microelectromechanical systems and new materials; in addition, we found the variety of potential applications appealing.

\section{Problem Definition}

The problem posed by the client consists of several requirements. The principal task is to design and build a robotic tentacle; a prototype consisting of at least two joints in series is required to demonstrate tethered operation (i.e. power and control delivered by wire). A digital model of at least ten joints in series is also requested.

The principal constraints are size (2" diameter) and cost ( $\$ 1000$ total project budget). A variety of performance criteria are specified: the tentacle should be able to go from straight to grasping under normal gravity in at most a few seconds. A power and control system is requested; it should scale easily with tentacle length.

A functioning roll degree of freedom (DOF) is optional; modular design is similarly optional. Mechanical design is top priority for the client, and electrical design is of secondary importance. Within the given budget, the prototype should consist of as many joints as possible. All design alternatives that are seriously considered have been documented.

\section{Design methodology}

Due to our budget constraints, and because of our client's expressed wish to have as many joints delivered as possible, we used standard parts from local suppliers wherever possible. This saved both cost and time; it also simplified the detailed design. We ordered from more distant suppliers when necessary, taking into account the related lead times. 
Over the course of the project, a number of decisionmaking criteria emerged as universally important. Taken together, the following considerations can be thought of as encapsulating our design philosophy.

In order to maximize tentacle performance, low weight was a priority for all components. This led, for example, to the selection of nylon fittings over those of aluminum or steel. As mentioned previously, cost was kept low to maximize the client's return, and off-theshelf parts were used wherever possible. These connect with another guiding principle: ease of use, which makes the client's long-term satisfaction more likely. Finally, one of the distinctive features of this project was the emphasis on self-contained mechanical, electrical, and control systems. As a result, our aim throughout was to produce a device that was both scalable and modular.

Our first step was to carry out a review of current research and identify similar areas of investigation. We also translated the hard project requirements (size constraint, performance criteria) into engineering targets; over the course of several meetings with the client, we identified and prioritized additional, "soft" requirements.

We then generated and evaluated a broad range of concepts, at both component and device levels. At this stage, we evaluated and discarded a number of possible functionalities. For simplicity, we chose not to pursue the development of a roll DOF; we similarly chose not to implement a joint elongation DOF. We identified the possibility of achieving the desired 2-DOF motion with only three actuators; however, the advantages of an arrangement consisting of two independent actuator pairs (additional force generation capacity, as well as simplicity of analysis) were thought to outweigh the weight savings and reduced range of spine/actuator interference of a three-actuator layout. In future, if more powerful, compact actuators can be developed, it may we worth reconsidering this choice.

We subsequently evaluated the available actuator candidates; geometrical and budget constraints eliminated all but one. Similarly, we considered a number of alternative component designs (end plate, spine and actuator attachment, in particular). These design alternatives were documented, as requested by the client.
In consultation with our advisors and in parallel with the ongoing mechanical design, we then began to develop a scalable power and control scheme. We identified a simple microchip that could be used to demultiplex and interpret control signals; given the project timeframe, however, and the tradeoff between immediate usability and long-term functionality, we felt the expense of implementing an inferior on-board control system was unjustified. We therefore decided to generate control signals in on a PC running Matlab (Simulink) and deliver them directly via the serial port.

While building the first joint prototype, a number of refinements immediately suggested themselves. In order to reduce weight and increase production values, we revised our choice of materials, machining and actuator attachment method for the end plates. Several practical improvements were made in the actuator assembly method as well (chiefly wire-wrapping techniques); these practices were largely the fruit of hands-on manufacturing experience.

At this point, we were able to assemble and demonstrate a fully-functional prototype joint. The mechanical operation of the joint was exhibited by implementing a simple, manually-switched relay control scheme; several further mechanical and electrical improvements resulted.

\section{Proposed solution}

The proposed joint consists of two roughly circular endplates connected by a rigid central spine. This spine is articulated by a universal joint located at the spine midpoint; this allows arbitrary plate deflection with respect to the geometrical center of the joint. Four linear actuators are connected to each plate via a ball-and-socket joint. Eight circular holes are cut out of the joint endplates to reduce weight and allow passage of power and control wiring (which span the entire length of the tentacle).

The joint end plates are made of $3 \mathrm{~mm}$ acrylic plate, as it is light weight and can be precisely machined with ease (in a laser cutter, for example). The actuators are pin-connected to the plate via a countersunk bolt at each end of the actuator; the bolt heads are nested in countersunk holes on opposite sides of each end plate, allowing the actuators independent pitch and yaw motion. In accordance with the general weight-saving philosophy, standard sized nylon fittings are used. 


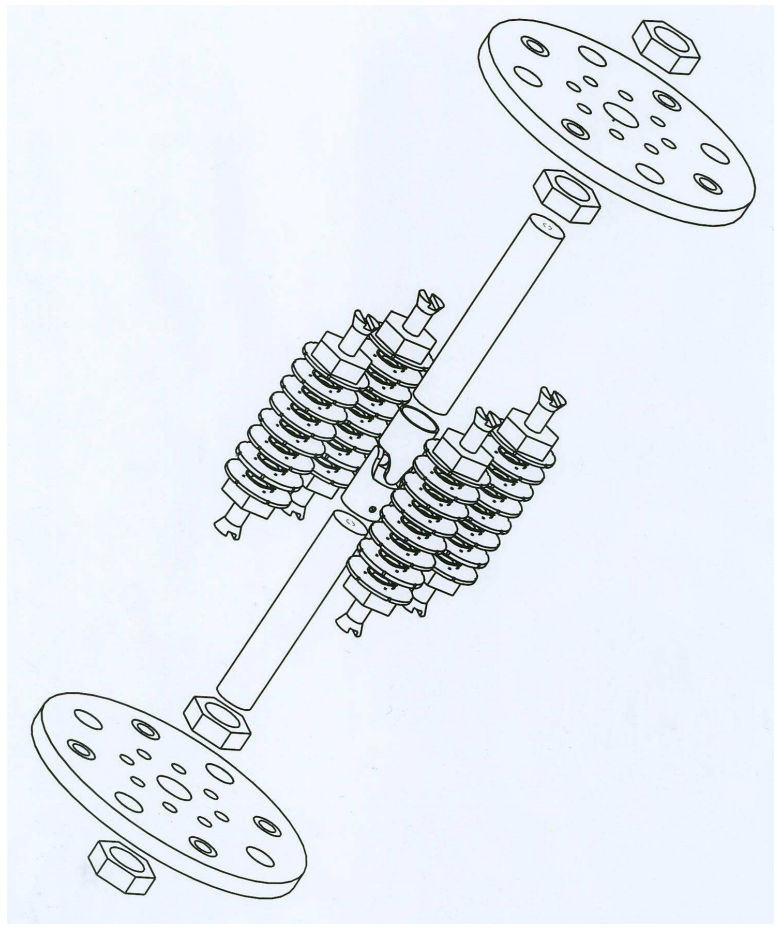

Figure 1. Single joint (exploded view)

The actuators of successive joints are offset by fortyfive degrees; thus, the actuators of every second joint are aligned. This configuration is motivated by the client's request for modularity and easy repair/replacement of actuators.

Joint angular displacement is accomplished by means of agonist/antagonist linear actuator pairs. We considered a number of candidate actuator technologies, but project constraints eliminated all but one. Conventional electric actuators, while inexpensive, are too large for the desired joint dimensions. Piezoelectric actuators are not capable of delivering a large enough stroke, while electroactuated polymers are too expensive and are thought to be an immature - and therefore risky - technology. Shape memory alloys (SMA), however, can be made to satisfy size, cost, and stroke requirements. In particular, the actuator design proposed by Grant in [1] and [2] overcomes SMA strain limitation and is generally well suited to the requirements of the project (with the caveat that the power draw per actuator is quite high).

Following Grant's work, we use the nickel-titanium alloy Nitinol, the most widely used SMA. The shape memory effect is a temperature-dependent phase transition which shows strong hysteresis behaviour. When heated above the transition temperature (approximately $90^{\circ} \mathrm{C}$ ), the alloy undergoes a Martensite-Austenite phase transition. Consequently, the wire length decreases and a tensile stress is developed - these two properties are exploited in the actuator design. Heat is generated by passing current through the wire; since the wire resistance is known, this heat addition can be accurately controlled. In order to ensure a useful life span, a 5\% strain limit must be imposed. By arranging the wire in a roughly helical configuration, the overall stroke is amplified considerably; design parameters include disc diameter, disc spacing and weave angle.

Our actuator consists of a series of stacked aluminum discs (diameter $20 \mathrm{~mm}$, thickness $0.007 \mathrm{~mm}$ ) held 5 $\mathrm{mm}$ apart by means of pretensioned springs. These discs contain six equally spaced semicircular notches (radius $0.002 \mathrm{~mm}$ ) along their circumference. A single strand of Flexinol wire is woven from notch to notch along the exterior of the actuator, passing twice through each of the notches to form a twelve-strand network. By delivering a current of approximately $2 \mathrm{~A}$ to this actuator, a stroke on the order of $20 \mathrm{~mm}$ is produced. The chosen low-gauge wire trades off large force generating potential for rapid cooling, which decreases the return stroke time once current is removed. The springs between discs maintain actuator integrity when not assembled in the joint; they also assist in the return stroke.

All actuators are connected in parallel to a $12 \mathrm{~V}$ DC power source. One drawback to this scheme is the constant, high current draw (approximately 4A per joint); for a tentacle of useful length, this will require the use of current amplifiers. Each joint consists of

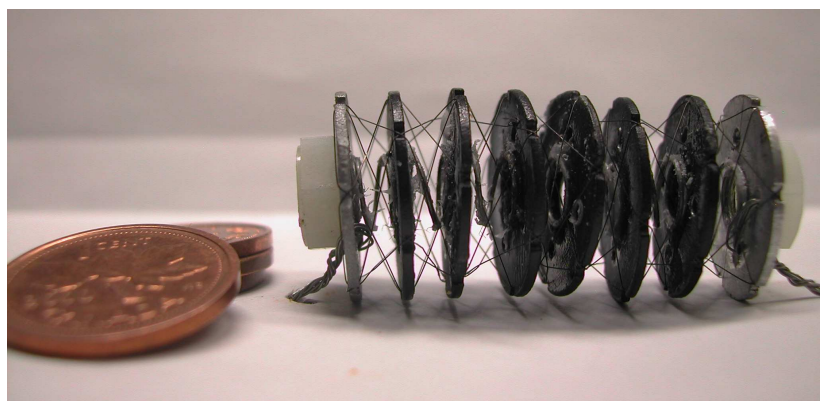

Figure 2. SMA linear actuator

two actuator pairs; only one member of each pair is active at any moment. The proposed control scheme sees power alternately switched between the members of each actuator pair; joint pitch or yaw is accomplished by independently varying duty cycles along the length of the tentacle.

Switching is accomplished by varying the base current to a transistor in series with each actuator. As discussed previously, these base currents are directly connected to the serial port of a PC. A more scalable solution would be a single control bus with microchips at each joint to demultiplex and execute control commands; this will have to wait for a future project cycle.

\section{Conclusion}

The joint design we have proposed fulfills the client's request for a control strategy test bed. The shape memory actuators perform as expected; tests carried 
out on the prototype joint reveal deflections of up to 30 degrees. Though we managed to keep the weight of the joint low through careful component selection, we recommend exploring a scheme where joint size tapers towards the tentacle tip; in this way, the tentacle length limit presently imposed by actuator force capacity can be exceeded.

The proposed control system adequately demonstrates tentacle joint capabilities. In a future project cycle we suggest implementing closed-loop control; to accomplish this, a feedback loop must incorporate real-time measurements of joint positions into the control algorithm. ShapeTape ${ }^{\mathrm{TM}}$ [2] is a likely candidate for such an operation. Furthermore, in order to achieve untethered operation, an embedded control system needs to be developed.

\section{References}

[1] D. Grant, "Shape Memory Alloy Actuator with an Application to a Robotic Eye," master's thesis, McGill University, 1995.

[2] D. Grant, "Accurate and Rapid Control of Shape Memory Alloy Actuators," Ph.D. thesis, McGill University, 1999.

[3] ShapeTapeTM is a fiber optic based 3D bend and twist sensor; it provides accurate real-time position and orientation information, and can be used to form real-time 3D computer images and collect data corresponding to complex shapes. Supplier: Measurand Inc.; for more details, see http://www.measurand.com/products/ShapeTape.html 